\title{
Perfil epidemiológico da mortalidade neonatal no Estado de Goiás de 2005 a 2015
}

\author{
Epidemiological profile of neonatal mortality \\ in the State of Goiás from 2005 to 2015
}

Heloisa Silva Guerra ${ }^{I}$ Bruna Abdo Pires ${ }^{\mathrm{I}}$

Heloisa Rodrigues SoARes da SILVA ${ }^{I}$

Larissa Mercadante de Assis ${ }^{1}$ LUISA SOUSA Eleutério de Azevedo ${ }^{1}$

Nathalya Chaves Meirelles ${ }^{I}$

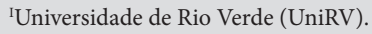
Aparecida de Goiânia/GO - Brasil
Resumo A mortalidade neonatal é definida como o óbito que ocorre nas quatro primeiras semanas de vida. Esse coeficiente é subdividido em mortalidade neonatal precoce, quando ocorre até o sexto dia de vida, e tardia, quando ocorre a partir do $7^{\circ}$. dia de vida. É um importante indicador de situação da população usado para planejar e desenvolver as políticas públicas de saúde. Este é um estudo descritivo epidemiológico, que teve o objetivo de analisar as principais causas que contribuem para os óbitos neonatais considerados evitáveis por adequada atenção ao recém-nascido no Estado de Goiás no período de 2005 a 2015. Para tal, foram utilizadas informações coletadas do banco de dados do Departamento de Informática do Sistema Único de Saúde (DATASUS), via Sistema de Informação de Mortalidade (SIM). Os resultados apresentados neste estudo evidenciam que a taxa de mortalidade infantil no Estado de Goiás tem diminuído, mas ainda constitui um problema de saúde, necessitando de ampliação do acesso aos serviços de pré-natal e acompanhamento puerperal da gestante.

Palavras-chave: Mortalidade Infantil. Mortalidade Neonatal Precoce. Mortalidade. Qualidade da assistência À saúde. PrÉ-NATAL.

Abstract Neonatal mortality is defined as the death that occurs in the first four weeks of life. This coefficient is subdivided into early neonatal mortality, when it occurs until the sixth day of life, and late, when it occurs from the 7th day of life. Is an important indicator of the population situation used to plan and develop public health policies. This is a descriptive epidemiological study that aimed to analyze the main causes that contribute to the neonatal deaths considered avoidable by adequate attention to the newborn in the state of Goiás from 2005 to 2015. To do so, we used information collected from the bank (DATASUS), via the Mortality Information System (SIM). The results presented in this study show that the infant mortality rate in the state of Goiás has decreased, but it still constitutes a health problem, necessitating an increase in access to prenatal services and puerperal follow-up of the pregnant woman.

Keywords: Infant Mortality. Early Neonatal Mortality. Mortality. Quality of health Care. Prenatal. 


\section{INTRODUÇÃo}

A mortalidade neonatal (MN) é definida como o óbito que ocorre nas quatro primeiras semanas de vida, ou seja, entre 0 e 27 dias completos após o nascimento. Esse coeficiente é subdividido em mortalidade neonatal precoce, quando acontece até o sexto dia de vida, e tardia, quando ocorre a partir do $7^{\circ}$. dia de vida. ${ }^{1}$

A MN é um componente da taxa de mortalidade infantil, um importante instrumento para avaliar a situação de saúde da população, sendo usado para planejar e desenvolver as políticas públicas de saúde. ${ }^{2}$ Sua redução faz parte das metas dos Objetivos de Desenvolvimento do Milênio 4, o qual visava uma redução de dois terços na taxa global de mortalidade de crianças com menos de 5 anos de idade entre 1990 e $2015 .^{3}$

Os relatórios mostraram que houve progresso na sobrevivência infantil, sendo que as taxas de mortalidade infantil no mundo caíram de 9,9 milhões, em 2000, para 5,6 milhões, em 2016, ou seja, uma redução de $43,4 \%$. Porém, quando se analisa os dados de acordo com o componente neonatal tem-se um crescimento da mortalidade. Nesse mesmo período, houve um aumento de $41 \%$ para $46 \%$ na mortalidade neonatal, o que significa o óbito de sete mil bebês a cada dia no mundo. Se não houver alguma intervenção efetiva, as previsões são que entre 2017 e 2030 mais 30 milhões de recém-nascidos morrerão nos primeiros 28 dias de vida. ${ }^{4}$

Além disso, a distribuição das mortes neonatais ocorre de maneira desigual no mundo, sendo o sul da Ásia e a África subsaariana responsáveis por quase $80 \%$ dessas mortes. Os países com maiores taxas de $\mathrm{MN}$ em ordem decrescente são: Índia, Paquistão, Nigéria, República Democrática do Congo e Etiópia. ${ }^{4}$

$\mathrm{Na}$ América Latina, entre o período de 1990 a 2016, houve um declínio de 59\% da mortalidade neonatal, atingindo a maior taxa de redução para a mortalidade neonatal mundial, nesse período. ${ }^{4}$ Dos países do continente americano, o Uruguai fica entre os cinco com os índices mais baixos com Canadá, Chile, Cuba e Estados Unidos. Entre 1990 e 2010, os países da região, com o apoio da Organização Pan-Americana da Saúde (OPAS) e parceiros, reduziram a mortalidade infantil para mais da metade. ${ }^{5}$

O Brasil é uma das nações que têm se destacado por reduzir a mortalidade infantil. Entre os anos de 1997 e 2012, houve uma queda de $33 \%$ na taxa de mortalidade neonatal precoce e uma redução de $21 \%$ na taxa de mortalidade neonatal tardia. Apesar dessa redução, o óbito neonatal constitui o principal componente da mortalidade infantil desde a década de 90, representando mais de $70 \%$ dos óbitos no primeiro ano de vida. ${ }^{6}$ Segundo o DATASUS, no Brasil entre os anos de 2000 até 2016, houve 540.349 mortes neonatais, sendo que 415.156 correspondem ao componente neonatal precoce, ou seja, $76,83 \%$ das mortes neonatais, evidenciando que o componente neonatal precoce continua sendo o principal fator do elevado nível de $\mathrm{MN}^{2}{ }^{2}$ A dificuldade em reduzir a $\mathrm{MN}$, principalmente a precoce, pode ser explicada pelo fato dessa fase ser a mais vulnerável da vida da criança, sendo influenciada não apenas por aspectos biológicos, mas também socioeconômicos relacionados às condições de saúde materna, qualidade na assistência do pré-natal e problemas relacionados ao parto. ${ }^{6}$ 
Sabendo da enorme influência que os fatores socioeconômicos geram na $\mathrm{MN}$, é de se esperar que a distribuição dos óbitos neonatais ocorra de forma desigual no Brasil. Isso foi demonstrado no estudo de Rodrigues et al., ${ }^{6}$ que avaliaram a mortalidade neonatal espacial e temporal no Brasil entre 1997 e 2012 e concluíram que a região nordeste e norte apresentou a maior taxa de MN do período estudado. ${ }^{6}$ Essa associação da distribuição geográfica com maiores taxas de óbito fetal também foi descrita em estudo realizado entre 2000 e 2007, concluindo que $50 \%$ dos óbitos de menores de 1 ano de idade ocorreram na região nordeste e na Amazônia legal (incluindo o Maranhão). ${ }^{7}$

$\mathrm{O}$ estudo mais recente sobre a mortalidade neonatal no Estado de Goiás foi realizado em $2000 .{ }^{8}$ Nesse estudo, o óbito neonatal representou $67,5 \%$ da mortalidade infantil. Os fatores de risco associados à mortalidade neonatal foram: nascer em hospital público-estatal, baixo peso ao nascer (menor de 2.500g), prematuridade (nascidos antes de 37 semanas completas) e nascidos vivos de gravidez múltipla. ${ }^{8} \mathrm{O}$ aspecto que mais chamou a atenção foi o fator nascer em hospital público-estatal, sendo possível analisá-lo como um marcador socioeconômico das famílias e no aspecto da qualidade da assistência prestada pelo serviço público. ${ }^{8}$

Portanto, a MN constitui-se como um sério problema de saúde pública, sendo fundamental conhecer o seu perfil em Goiás com a finalidade de gerar subsídios para a criação e implementação de políticas públicas eficazes e, assim, melhorar a qualidade de vida dos neonatos. Nesse sentido, este estudo teve como objetivo analisar as prin- cipais causas que contribuem para os óbitos neonatais considerados evitáveis por adequada atenção ao recém-nascido no Estado de Goiás no período de 2005 a 2015.

\section{Material e Métodos}

Trata-se de um estudo descritivo epidemiológico com fontes de dados secundários coletados do banco de dados do Departamento de Informática do Sistema Único de Saúde (DATASUS), via Sistema de Informação de Mortalidade (SIM).

Foram descritos os óbitos neonatais (0 a 27 dias de vida) por causas evitáveis por adequada atenção ao recém-nascido por local de residência, ocorridos no período de 2005 a 2015 em Goiás. Entende-se como evitabilidade do óbito aqueles que poderiam ser evitados ou não deveriam ter ocorrido, considerando as ciência e tecnologias existentes. Incluem-se nesse grupo as seguintes causas: transtornos respiratórios específicos do período neonatal (CIDs P22, P23, P25, P27 e P28); infecções específicas do período neonatal, (CIDs P35 - P39); hemorragia neonatal, exceto intracraniana não traumática (CIDs P50, P51, P53 e P54); outras icterícias neonatais (CIDs P58 e P59); transtornos endócrinos e metabólicos transitórios específicos do feto e do recém-nascido (CIDs P70 - P72 e P74); outros transtornos hematológicos do feto e do recém-nascido (CIDs P60 e P61); transtornos do aparelho digestivo do feto ou do recém-nascido, exceto enterocolite necrotizante (CIDs P75, P76 e P78); afecções que comprometem o tegumento e a relação térmica do feto e do recém-nascido (CIDs P80, P81 e P83) e outros transtornos originados no período 
perinatal (CIDs P90 - P94 e P96). Entende-se por óbito por local de residência aqueles ocorridos contados segundo o local de residência do morto.

Analisou-se a causa básica, evitabilidade do óbito, sexo, idade e óbitos por município de residência. A idade foi subdividida em mortalidade precoce de 0 a 6 dias, e tardia de 7 a 27 dias.

Por se tratar de dados de domínio público, o estudo não foi submetido ao Comitê de Ética e Pesquisa (CEP), porém, todos os princípios éticos para a realização da pesquisa foram respeitados. Os resultados encontrados são apresentados de forma descritiva.

\section{RESUltados}

Entre 2005 e 2015, segundo o SIM, foram registrados 2.505 óbitos neonatais por causas evitáveis relacionadas à adequada atenção ao recém-nascido no Estado de Goiás de acordo com as categorias CID-10 selecionadas. Entre as cidades que apresentaram maiores registros de óbitos se destacaram Goiânia, Aparecida de Goiânia, Anápolis e Rio Verde (Gráfico 1), coincidindo com as cidades de maior número populacional do Estado. Assim, essas quatro cidades são responsáveis por $999(39,88 \%)$ casos de mortalidade neonatal, enquanto o restante das cidades do Estado de Goiás correspondeu a 1.506 casos $(60,11 \%)$ por local de residência.

Gráfico 1: Óbitos neonatais por causas evitáveis por adequada atenção ao recém-nascido no Estado de Goiás, no período de 2005-2015.

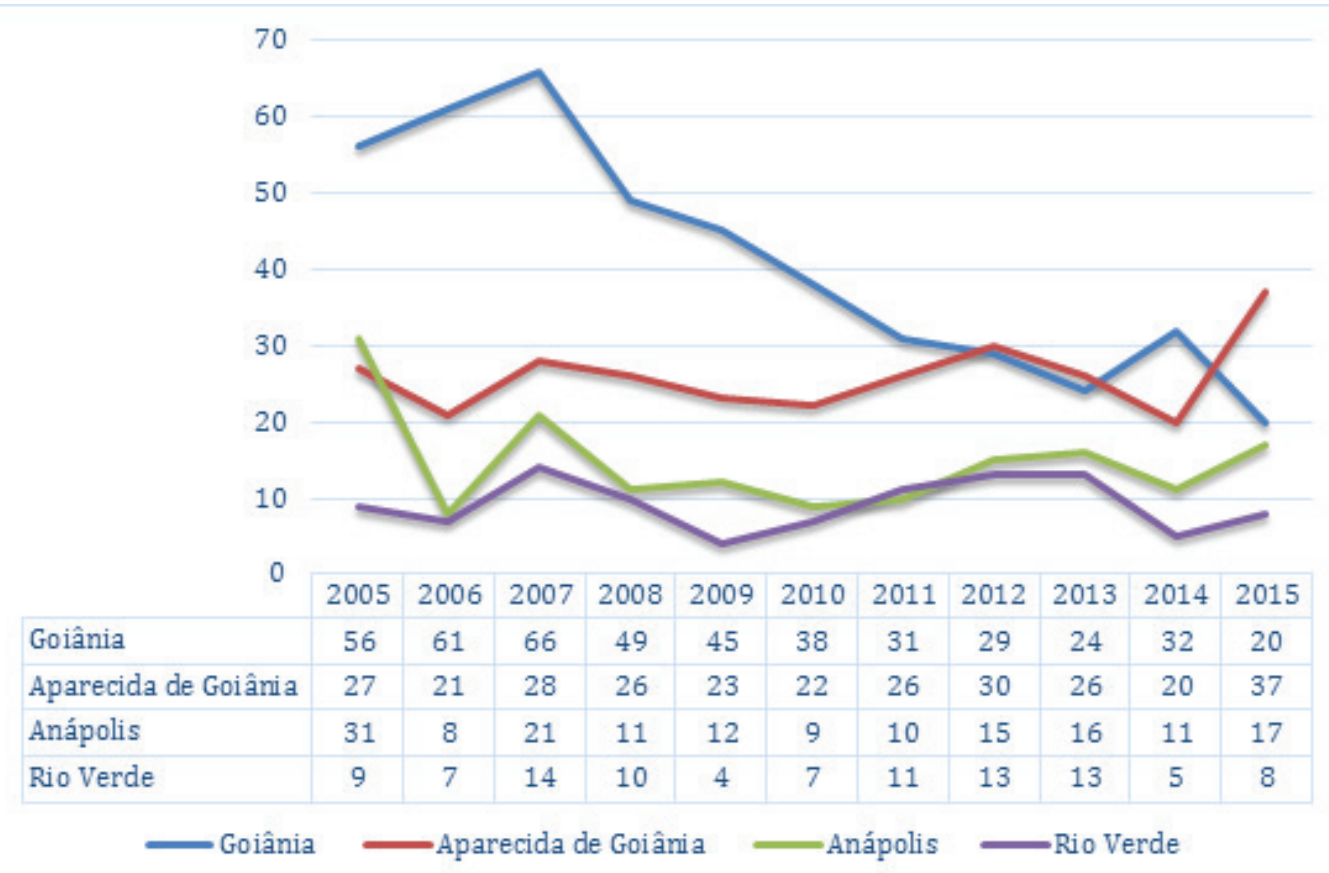

Fonte: Sistema de Informações sobre Mortalidade (SIM), 2018. 
Observou-se que Goiânia foi a cidade com a maior redução de óbitos neonatais ao longo desses dez anos, com a ocorrência de 56 (12,41\%) óbitos por local de residência em 2005 e de 20 (4,43\%) óbitos em 2015, representando uma redução de 36 (64,28\%) óbitos. Já em Aparecida de Goiânia, os óbitos permaneceram mais estáveis, não ultrapassando a variação de oito óbitos no período estudado, tendo como exceção o ano de 2015, no qual foram observados 37 óbitos, o que significa um aumento de 17 óbitos (45,94 \%) com relação ao ano anterior. Na cidade de Anápolis, a redução foi considerável de 2005 para 2006, passando de $31(19,25 \%)$ para oito $(4,69 \%)$ óbitos, porém, posteriormente manteve uma pequena oscilação nesses dez anos, com uma redução de 14 óbitos $(45,16 \%)$ de 2005 para 2015. Finalmente, Rio Verde foi a cidade que apresentou a menor redução de óbitos ao longo desses anos, mantendo em 2015, 9 (9,57\%) óbitos, próximo da quantidade encontrada em 2005, oito $(8,51)$ óbitos, diferenciando apenas por um caso.

A partir dos dados, observou-se que os números de óbitos neonatais precoces, que corresponderam a $1.556(62,11 \%)$ óbitos, são maiores que os neonatais tardios, cujos valores são 949 óbitos (37,88\%) (Gráfico 2). Além disso, foi possível verificar que o número de óbitos da categoria de 0 a 6 dias sofreu maior redução entre 2005 e 2015, correspondendo a $25,26 \%$, do que a categoria de 7 a 27 dias, correspondendo a 9,90\%.

Gráfico 2: Óbitos neonatais precoces e tardios por causas evitáveis por adequada atenção ao recém-nascido no Estado de Goiás, no período de 2005-2015.

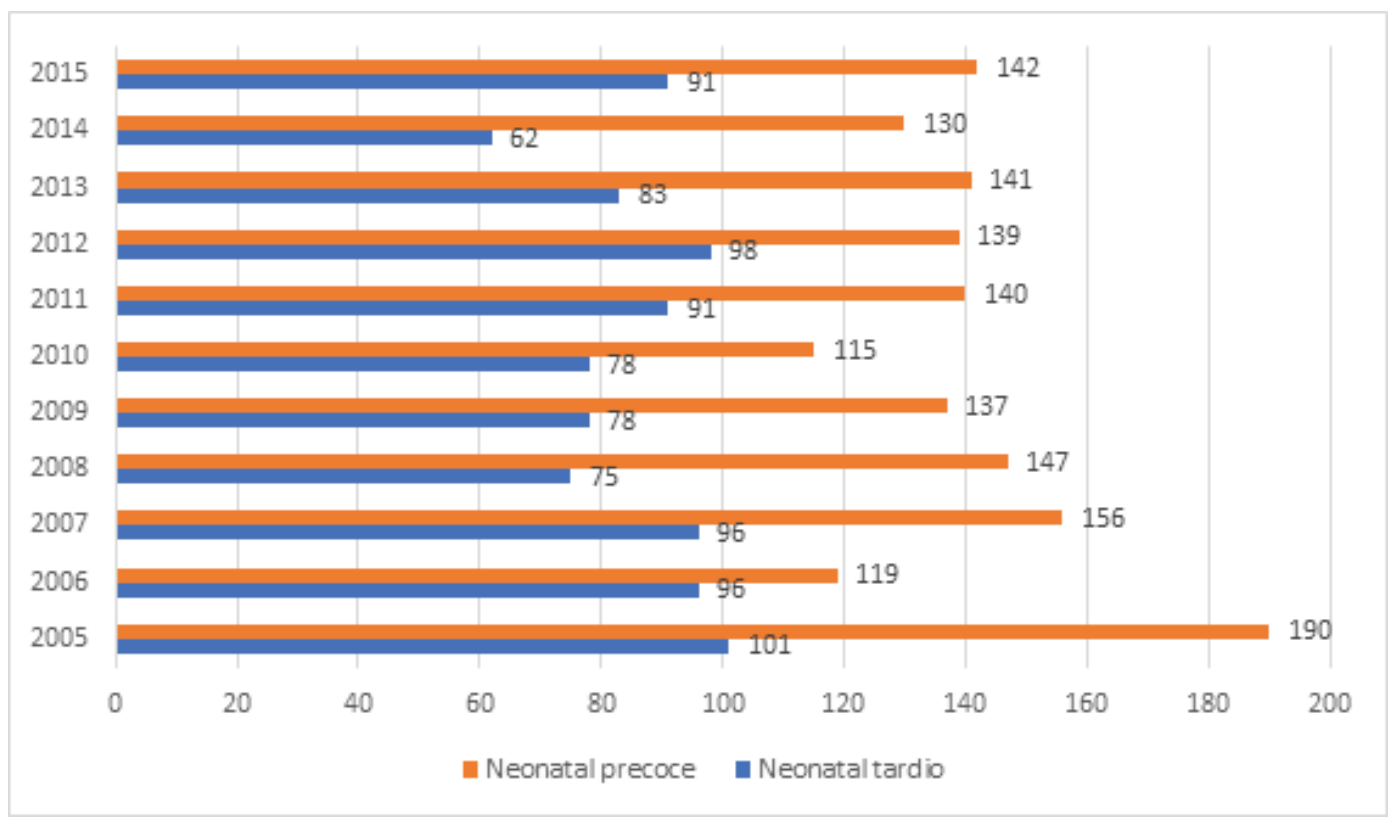

Fonte: Sistema de Informações sobre Mortalidade (SIM), 2018. 
De acordo com o Gráfico 3, foi possível notar que entre as principais causas evitáveis de óbitos reduzíveis por adequada atenção ao recém-nascido se destacaram três: as infecções específicas do período neonatal com 1.487 (59,36\%) óbitos, que incluem doenças virais congênitas (CID P35), septicemia bacteriana do recém-nascido (CID P36), outras doenças infecciosas e parasitárias congênitas (CID P37), onfalite com ou sem hemorragia leve (CID P38) e outras infecções específicas do período perinatal (P39); seguido dos transtornos respiratórios específicos do período neonatal responsável por 668 (26,66\%) óbitos, sendo pertencentes a esse grupo as ca- tegorias desconforto respiratório do recém-nascido (CID P22), pneumonia congênita (CID P23), enfisema intersticial originado no período perinatal (CID P25), doença respiratória crônica não especificada originada no período perinatal (CID P27) e outras afecções respiratórias de origem perinatal (CID P28); e o terceiro grupo mais predominante foi o das afecções que comprometem o tegumento e a regulação térmica com 145 $(5,78 \%)$ óbitos, sendo incluídas a hipotermia do recém-nascido (CID P80), outros distúrbios da regulação térmica (CID P81) e outras afecções comprometendo o tegumento específicas do feto e do recém-nascido (CID P83).

Gráfico 3: Principais causas evitáveis de óbitos reduzíveis por adequada atenção ao recém-nascido no Estado de Goiás, no período de 2005-2015.

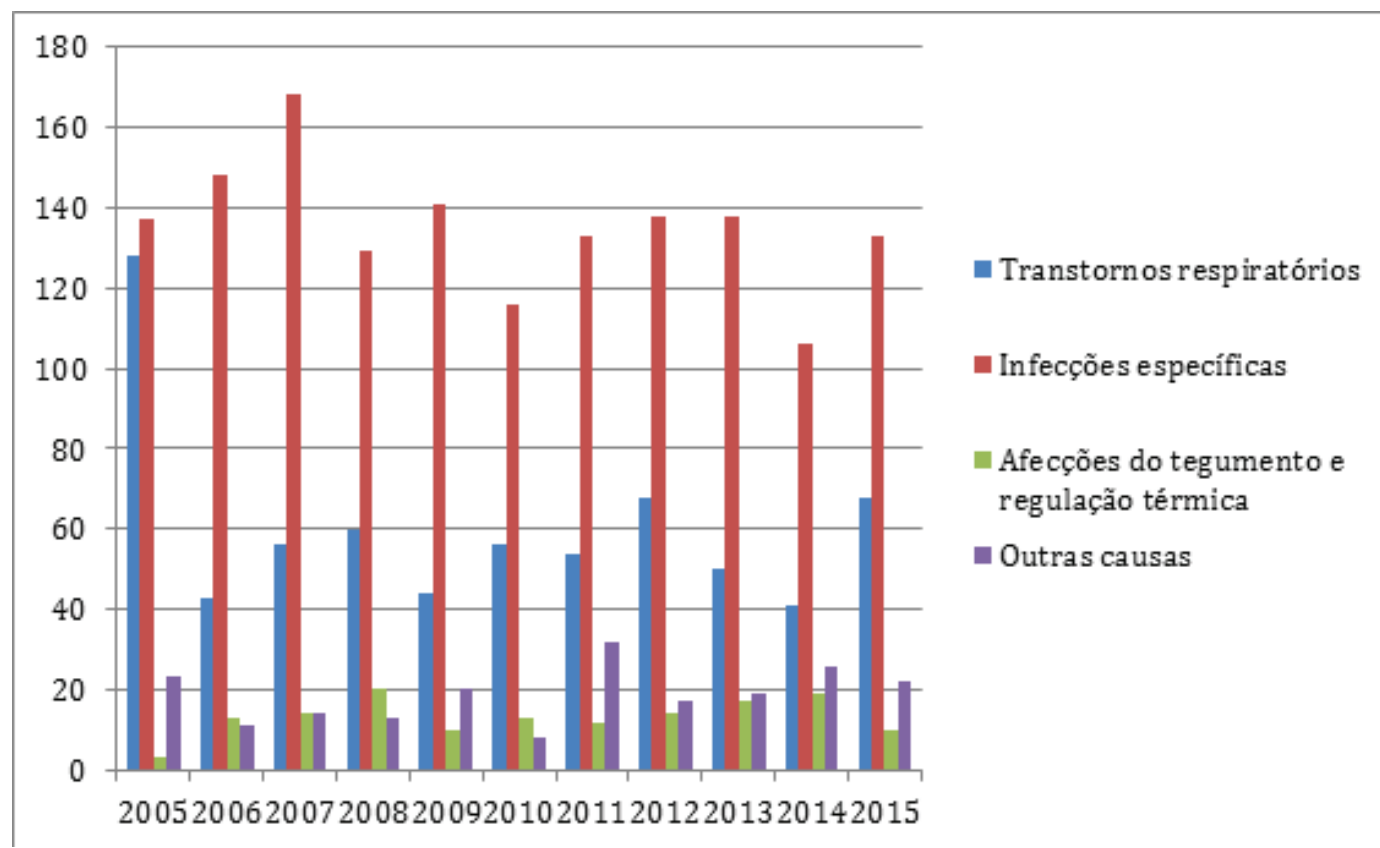

Fonte: Sistema de Informações sobre Mortalidade (SIM), 2018. 
As outras causas representadas pela hemorragia neonatal, exceto intracraniana não traumática (CIDs P50-P51,P53-P54); outras icterícias neonatais (CIDs P58-P59); transtornos endócrinos e metabólicos transitórios específicos do feto e do recém-nascido (CIDs P70-P72,P74); transtornos do aparelho digestivo do feto ou do recém-nascido, exceto enterocolite necrotizante (CIDs P75-P76,P78) e outros transtornos originados no período perinatal (CIDs P90 - P94 e P96), corresponderam juntas a 205 $(12,11 \%)$ casos de morte neonatal por local de residência durante o período analisado.

\section{DISCUSSÃo}

O componente neonatal é o principal representante dos óbitos em crianças antes de 1 ano de vida. Esse dado evidencia a importância dos fatores ligados ao pré-natal, parto, puerpério e assistência neonatal para a redução da mortalidade na infância. Sendo assim, a mortalidade neonatal é um indicador sensível da adequação da assistência obstétrica e neonatal e do impacto de programas de intervenção nessa área. ${ }^{9}$

Ao analisarmos os dados de mortalidade neonatal, encontramos um valor de 70,4\% das mortes neonatais, no Estado de Goiás, com um valor absoluto de 9.865 mortes até 27 dias de vida. ${ }^{2}$ Um número bastante expressivo, considerando um total de 13.745 mortes totais antes de um ano avaliadas no período de 2005-2015. ${ }^{2}$ A mortalidade infantil, em Goiás, diminuiu de 13,86 por mil nascidos vivos em 2013, para 12,84 por mil nascidos vivos, em 2014. Com isso, Goiás ocupava, em 2016, a $12^{\text {a }}$ posição do ranking dos Estados e se encontrava abaixo da Taxa Nacional de 12,89 por 1.000 nascidos vivos. ${ }^{10}$
De acordo com os dados oficiais do $\mathrm{Mi}$ nistério da Saúde (MS), em 2013 o Estado de Goiás apresentou 1.314 óbitos infantis, sendo que $70 \%$ desses foram de óbitos neonatais. A maioria desses óbitos ocorreu por causas evitáveis, como: prematuridade, pneumonia, complicações durante o trabalho de parto e diarreia, ou seja, causas evitáveis por ações efetivas dos serviços de saúde. ${ }^{11}$

Um estudo realizado em Novo Hamburgo, que avaliou as causas de mortalidade infantil no período de 2007 a 2010, encontrou resultado semelhante, apresentando $66,9 \%$ de óbitos no período neonatal, dos quais $77 \%$ ocorreram por causas evitáveis. ${ }^{12}$

No presente estudo, ocorreram 2.505 óbitos neonatais por causas evitáveis por adequada atenção ao recém-nascido no período estudado. Desses, os óbitos neonatais precoces corresponderam a $1.556(62,11 \%)$ casos, quantidade maior que os neonatais tardios, cujos valores foram de 949 óbitos (37,88\%). A redução foi maior no componente neonatal precoce, se comparado com o tardio.

As intervenções necessárias para enfrentar as principais causas de mortes no período neonatal diferem das utilizadas em outras faixas etárias infantis. Além disso, a sua diminuição é mais lenta que a observada nas taxas de mortalidade em crianças mais velhas, fatores que justificam uma atenção maior voltada para esse componente da mortalidade infantil. ${ }^{13}$

Apesar de observarmos uma queda significativa do componente neonatal precoce $(25,26 \%)$, a redução do componente tardio $(9,9 \%)$ não foi tão expressiva, com uma diminuição menor que 10\%. Essa redução dos óbitos neonatais precoce no Estado de Goiás pode ser atribuída à implantação de iniciativas governamentais, como o "Pro- 
grama Siga Bebê", que promove um acompanhamento do recém-nascido até 1 ano de vida. Além desse programa, as políticas nacionais, como a Rede Cegonha, implantada em 2011, também interferiram na redução desses números. Com relação à menor redução do componente tardio, pode-se associar com a melhora da atenção ao cuidado do recém-nascido nos primeiros sete dias de vida, levando à postergação de vida do neonato, então uma morte precoce se torna tardia. Ainda assim, é relevante questionar a abrangência desses programas, devido à importância de se melhorar as estatísticas de óbito do componente neonatal tardio. ${ }^{14}$

$\mathrm{O}$ acompanhamento rigoroso durante o pré-natal permite a identificação e intervenção precoce, evitando possíveis complicações futuras para o recém-nascido. Sendo assim, a garantia de assistência pré-natal de qualidade adequadamente conduzida e a organização da assistência em sistemas hierarquizados e regionalizados, de forma a garantir acessibilidade à gestante, podem detectar doenças maternas e fetais, melhorando assim a possibilidade de sobrevivência do recém-nascido e reduzindo a prevalência de restrição do crescimento intrauterino, a prematuridade e a ocorrência de baixo peso ao nascer. ${ }^{15}$

Entre as cidades do Estado de Goiás que exibiram maiores registros de óbitos (Goiânia, Aparecida de Goiânia, Anápolis e Rio Verde), estão as cidades de maior número populacional do Estado. No entanto, esses dados não comprovam uma pior qualidade da assistência à saúde ou escassez de recursos nessas cidades, considerando que normalmente as regiões com maior índice populacional apresentam os grandes centros de saúde e acabam acolhendo também situações de alto risco dos municípios vizinhos.

Outro fator que pode explicar o maior número de óbitos nas cidades mais populosas é a dificuldade de integrar a atenção primária com a secundária. A relação entre atenção primária e secundária não é adequada, ou muitos casos acabam chegando a esses municípios pelo fato de serem referência para os demais da região ou possuírem UTI neonatal pelo SUS. O modelo de atendimento prestado somente por unidades básicas de saúde talvez não comporte a captação da demanda de gestantes para a realização das consultas de pré-natal, o que é facilitado quando o município tem um modelo de atenção à saúde da família. A Estratégia de Saúde da Família (ESF) amplia o acesso e facilita a adesão do usuário, aumentando a resolutividade e a qualidade do serviço prestado à população. ${ }^{16}$

Assim, o aumento de cobertura e da qualidade da assistência ofertada pela Estratégia de Saúde da Família (ESF) pode melhorar o preparo da mulher para a gravidez, aumentar o intervalo intergestacional, reduzir intercorrências na gestação, melhorar a detecção do diabetes e hipertensão gestacionais e pré-eclâmpsia, melhorar a continuidade do cuidado envolvendo maternidades, o que globalmente reduz a mortalidade perinatal. ${ }^{16}$

As desigualdades sociais entre regiões e populações no Brasil se reproduzem nas taxas de mortalidade infantil, constituindo um relevante problema de saúde a ser enfrentado por toda a sociedade. Outros estudos ${ }^{6,9,15}$ demonstraram que a mortalidade neonatal é maior nos locais com piores condições socioeconômicas. Os dados mais recentes divulgados pela Secretaria de Saú- 
de do Estado de Goiás evidenciam também uma desigualdade na distribuição de recursos. Observou-se que $12 \%$ dos municípios de menor infraestrutura concentram $76 \%$ da mortalidade infantil e $70 \%$ das mortes são de neonatais. ${ }^{10}$

O fator socioeconômico interfere também nas causas dos óbitos neonatais. No Brasil, prevalecem como causas de mortalidade perinatal a asfixia intrauterina e intraparto, o baixo peso ao nascer, as afecções respiratórias do recém-nascido, as infecções e a prematuridade, diferindo-se dos países desenvolvidos, onde a prematuridade extrema e as malformações congênitas, mortes que não se pode prevenir, são as principais causas de óbito. ${ }^{17}$

$\mathrm{Na}$ realidade brasileira, a classificação mais aplicada em estudos de mortalidade neonatal é a proposta pela Fundação Sistema Estadual de Análise dos Dados de São Paulo (Seade), que agrupa as causas de óbito segundo critérios específicos de evitabilidade. Nessa proposta, as mortes peri e neonatais evitáveis são organizadas em três grandes agrupamentos: mortes evitáveis por adequado controle da gravidez, por adequada atenção ao parto e por adequada atenção ao recém-nascido. ${ }^{18}$

Uma limitação dessa classificação para a realidade brasileira se refere à relação direta das mortes perinatais evitáveis com a assistência obstétrica e neonatal, ou seja, a assistência clínica. No Brasil, o acesso aos serviços de saúde em tempo oportuno ainda é um problema, sendo frequente a peregrinação das gestantes até a sua internação definitiva e mesmo a sua internação já em período expulsivo, o que se torna fator determinante do resultado para a criança, pois diminuem as chances de intervenções clínicas eficazes. ${ }^{19}$

No presente estudo, as infecções específicas do período neonatal, como sífilis, toxoplasmose, rubéola, citomegalovirose, herpes e HIV foram responsáveis por 1.487 $(59,46 \%)$ óbitos neonatais. Essas causas estão relacionadas com a prematuridade, baixo peso ao nascer e malformações congênitas, indicando a possibilidade de precariedade do pré-natal, pois poderiam ser evitadas se o acompanhamento da gestante fosse realizado adequadamente.

É importante destacar que um pré-natal de qualidade está diretamente relacionado ao bem-estar materno-infantil, pois o acompanhamento não adequado durante o pré-natal acarreta graves consequências ao binômio mãe-filho, por exemplo, infecções do trato geniturinário recorrentes, prematuridade e o baixo peso ao nascer, sendo estes os principais fatores de risco para a sepse neonatal. ${ }^{20}$

A segunda maior causa de óbito neonatal foram os transtornos respiratórios específicos do período neonatal, responsáveis por 668 (26,66\%) óbitos no período estudado. A maioria das doenças respiratórias neonatais se manifesta nas primeiras horas de vida, de forma inespecífica e, muitas vezes, com sobreposição de sinais e sintomas. ${ }^{21}$ Isso demonstra a importância da presença de profissionais capacitados, para identificarem e atuarem sobre tais distúrbios o mais precocemente possível. Além dos profissionais, as mães e familiares também precisam ser orientados quanto às alterações que podem sugerir risco de algum distúrbio respiratório no recém-nascido, para que procurem o atendimento médico em tempo hábil. 
Estudo realizado em Cuiabá-MS observou a inversão desse padrão com os transtornos respiratórios e cardiovasculares específicos do período perinatal, correspondendo a cerca de um terço dos óbitos (35\%), seguidos dos óbitos por infecção específica do período perinatal $(21,6 \%) .^{22}$

O terceiro grupo mais prevalente foi o das afecções que comprometem o tegumento e a regulação térmica, com 145 (5,78\%) registros de óbitos, os quais podem tanto ser causa como consequência de doenças infecciosas, cardiovasculares, respiratórias, assim como as outras causas representadas pela hemorragia neonatal, exceto intracraniana não traumática. Dessa forma, a fisiopatologia e etiologia das categorias citadas acabam se misturando, mostrando uma falha na classificação. O que leva a uma análise de dados deficientes, pois não se pode identificar uma causa específica, visto a dificuldade de separar estas de sintomas ou patologias.

Outra causa de óbito neonatal de grande importância é a asfixia intraparto, que tem sido apontada como a causa de óbito com maior potencial de prevenção, se houver máxima supervisão durante o trabalho de parto. O óbito fetal ou neonatal por asfixia intraparto tem sido considerado uma medida sensível da qualidade da assistência durante o momento do pré-parto e nascimento. A asfixia aparece como terceira ou quarta causa na maioria dos Estados brasileiros, porém no Maranhão desponta como segunda causa. Essas mortes, por asfixia intraparto, poderiam ser reduzidas em $36 \%$ se houvesse uma melhor assistência ao parto e ao nascimento. ${ }^{9}$

Muitas medidas já foram tomadas na tentativa de amenizar os desfechos já ana- lisados. Foram implementadas nos municípios goianos desde 2013, a Atenção Integrada às Doenças Prevalentes na Infância - Componente Neonatal, que fornece a médicos e enfermeiros da Atenção Básica conhecimentos para a detecção precoce e tratamento efetivo das principais doenças que afetam a saúde das crianças menores de 5 anos de idade. E, desde o ano de 2014, a Gerência de Saúde iniciou um programa de capacitação em Reanimação e Transporte Neonatal para todos os médicos e enfermeiros das Unidades de Suporte Avançadas dos Serviços de Atendimento Móvel de Urgência (SAMU) dos municípios que transportam recém-nascidos às principais maternidades do Estado. ${ }^{23}$

Ainda, em 2015 foi lançado, nas cinco macrorregiões de saúde do Estado de Goiás, o Programa Mais Saúde para Goiás, que conta com seis projetos de fortalecimento da atenção primária à saúde nos municípios goianos, como o aumento do número de Equipes de Saúde da Família e equipamentos de apoio diagnóstico aos municípios. Como parte do programa, no período de 2015 a 2017 foram realizadas seis oficinas temáticas e conceituais para a qualificação dos profissionais da Estratégia Saúde da Família (ESF) e suas equipes multiprofissionais que atuam na APS nos 246 municípios, organizada em um processo de educação permanente. As oficinas abordaram os temas: As Redes de Atenção à Saúde; A Atenção Primária à Saúde no Município; Territorialização; Vigilância em Saúde; e Organização da UBS e do Processo de Trabalho em Saúde. ${ }^{24}$

Este estudo apresentou algumas limitações relacionadas à fonte de dados secundários, que estão sujeitos à subnotificação, uma 
realidade ainda presente principalmente nos municípios mais afastados. Além disso, os dados coletados não foram confrontados com os relatórios gerenciais.

\section{CONSIDERAÇões FINAIS}

A mortalidade infantil é considerada um importante problema de saúde pública e é um forte indicador de situação de saúde da população. Os resultados apresentados neste estudo evidenciaram que a mortalidade neonatal em Goiás tem diminuído, mas ainda constitui um problema de saúde pública. Isso se dá pelo aprimoramento quanto à qualidade dos cuidados imediatos após o nascimento, da assistência no pré-natal, intraparto e as condições de assistência ao parto e pós-parto. Além disso, outros fatores como baixo peso ao nascer, prematuridade, nascer em hospital público e gravidez múltipla influenciam diretamente na assistência ao recém-nascido, impactando diretamente na taxa de mortalidade infantil.

Sendo assim, faz-se necessária uma ampliação do acesso aos serviços públicos de saúde com um adequado acompanhamento pré-natal da gestante, com o objetivo de detectar precocemente doenças maternas e fetais e uma possível intervenção no momento adequado, além de uma assistência apropriada ao trabalho de parto, parto e pós-parto, mantendo-se a continuidade do cuidado ao recém-nascido e à puérpera.

\section{REFERÊNCIAS}

1. Brasil. Ministério da Saúde. Rede Interagencial de Informações para a Saúde [Internet]. Características de indicadores: Fichas de qualificação, Mortalidade; 2012 [cited 2012 Sept 12]. Available from: http://www.ripsa.org.br/fichasIDB/level.php?node=C\&lang=pt\&version=ed5

2. Datasus. Mortalidade [Internet]. Brasília (DF): Ministério da Saúde; 2018 [citado 2018, mai. 18]. Disponível em: http://tabnet.datasus.gov.br/cgi/tabcgi.exe?sim/cnv/obt10uf.def

3. Gaffey MF, Das JK, Bhutta ZA. Millennium Development Goals 4 and 5: past and future progress. Semin Fetal Neonatal Med. 2015, Oct; 20 (5): 285-92.

4. Hug L., Sharrow D., You D. Levels \& trends in child mortality: report 2017. Estimates developed by the UN inter-agency Group for Child.

5. Nações Unidas do Brasil. Saiba como o Uruguai reduziu a mortalidade infantil a um mínimo histórico em 20 anos [Internet]. Brasil: Nações Unidas, 2018 [atualizada em 2018, abr. 13]. Disponível em: https://nacoesunidas.org/saiba-como-o-uruguai-reduziu-a-mortalidade-infantil-a-um-minimo-historico-em-20-anos/

6. Rodrigues NC, Monteiro DL, Almeida AS, Barros MB, Pereira Neto A., O'Dwyer G., et al. Temporal and spatial evolution of maternal and neonatal mortality rates in Brazil, 1997-2012. J Pediatr. 2016, nov./dez.; 92 (6): 567-573.

7. Oliveira GS, Lima MCBM, Lyra CO, Oliveira AGRC, Ferreira MAF. The spatial inequality of neonatal mortality in Brazil: 2006 to 2010. Ciênc. saúde coletiva. 2013, Aug.; 18 (8): 2.4312.441 .

8. Associação Goiana de Municípios. Mortalidade infantil diminui em Goiás, mas 30 municípios precisam melhorar índices [Internet]. Goiás, 2016 [atualizada em 2016, jul. 21]. Disponível em: http://www.agm-go.org.br/noticia/337-mortalidade-infantil-diminui-em-goias-mas-30-municipios-precisam-melhorar-indices 
9. França EB, Lansky S., Rego MAS, Malta DC, França SJ. Principais causas da mortalidade na infância no Brasil, em 1990 e 2015: estimativas do estudo de Carga Global de Doença. Rev Bras Epidemiol. 2017, mai. 20 (1): 46-60.

10. Datasus. Informações de saúde. [Internet]. Brasil: Ministério da Saúde; 2016 [acesso em 2019, jun. 2]. Disponível em: http://tabnet.datasus.gov.br/cgi/tabcgi.exe?sim/cnv/fet10go.def

11. Ministério da Saúde. Banco de dados dos Sistemas de Informação sobre Mortalidade (SIM), 2013. Estatística e informação em saúde. Brasília: Secretaria de Vigilância em Saúde, Departamento de Análise de Situação em Saúde; 2018.

12. Brum CA, Stein AT, Pellanda LC. Mortalidade Infantil em Novo Hamburgo: Fatores Associados e Causas Cardiovasculares. Arq Bras Cardiol. 2015; 104 (4): 257-265.

13. Unicef. Fundo das Nações Unidas para a Infância. Statistics by area: child survival and health: under five mortality. 2015.

14. Goiás. Secretaria de Estado De Goiás. Portaria no $\mathbf{4 5 1}$, de 2 de julho de 2016. Criando o grupo condutor dos Programas Siga Bebê e Mais Saúde para Goiás. Superintendência De Gestão, planejamento e finanças. 2016, jun. 2; Seção 1, p. 3.

15. Nascimento RM, Leite AJM, Almeida NMG S., Almeida PC, Silva CF. Determinantes da mortalidade neonatal: estudo caso-controle em Fortaleza, Ceará, Brasil. Cad. Saúde Pública. 2012, mai. 28 (3): 559-572.

16. Brasil. Ministério da Saúde. Secretaria de Vigilância em Saúde. Secretaria de Ciência, Tecnologia e Insumos Estratégicos. Síntese de evidências para políticas de saúde: mortalidade perinatal. Brasília: Ministério da Saúde, 2016.

17. Fernandes CA, Vieira VCL, Scochi M. Mortalidade Infantil e classificação de evitabilidade: pesquisando municípios da 15 regional de saúde Paraná. Cienc Cuid Saude. 2013, out./dez.; 12 (4): 752-759.

18. Fundação Sistema Estadual de Análise dos Dados de São Paulo (Seade). Mortalidade Perinatal no Estado de São Paulo: Resenha de Estatísticas Vitais do Estado de São Paulo. 2011, fev.; (11) 2:1-9.

19. Martins MCF. Demoras na assistência obstétrica de gestantes com desfecho de óbito fetal (ante ou intraparto): estudo de caso-controle. [Dissertação]. Ceará: Universidade Federal do Ceará. 2018. Acesso em: 2019, jun. 2. Disponível em: http://brasil.evipnet.org/wp-content/uploads/2016/07/Mortalidade_perinatal_WEB_jul.pdf

20. Moreira KFA, Bicalho BO, Santos LCS, Siqueira FMGA, Órfão NH, Cunha MPL. Perfil e evitabilidade de óbito neonatal em um município da Amazônia legal. Cogitare Enferm. 2017, mai. (22) 2: 48950.

21. Brasil. Ministério da Saúde. Secretaria de Atenção à Saúde. Departamento de Ações Programáticas Estratégicas. Atenção à saúde do recém-nascido: guia para os profissionais de saúde / Ministério da Saúde, Secretaria de Atenção à Saúde, Departamento de Ações Programáticas Estratégicas. - 2. ed. - Brasília: Ministério da Saúde, 2012.

22. Gaiva MAM, Fujimori E., Sato APS. Fatores de risco maternos e infantis associados à mortalidade neonatal. Texto Contexto Enferm. 2016, jun.; 25 (4): 2-9.

23. Secretaria de Estado da Saúde. Superintendência de Gestão, Planejamento e Finanças. Relatório Anual de Gestão. 2015, mar., p. 60.

24. Oliveira ALI, Gomides EFC, Souto R., Santos TGM. A planificação da Atenção à Saúde no Estado de Goiás. Revista Consensus, 2019 (30). 


\section{DAdOS DAS AUTORAS}

\section{Heloisa Silva Guerra}

Mestre em Saúde Coletiva pela Universidade Federal de Goiás (UFG). Doutoranda em Saúde Coletiva pela Universidade do Vale do Rio dos Sinos (UNISINOS). Professora Adjunto I da Faculdade de Medicina na Universidade de Rio Verde (UniRV). Aparecida de Goiânia/ GO - Brasil. heloisasguerra@gmail.com

\section{Bruna Abdo Pires}

Graduanda de Medicina na Universidade de Rio Verde (UniRV). Aparecida de Goiânia/ GO - Brasil. brunaabdo@gmail.com

\section{Heloisa Rodrigues Soares da Silva}

Graduanda de Medicina na Universidade de Rio Verde (UniRV). Aparecida de Goiânia/ GO - Brasil. heloisa300494@gmail.com

\section{Larissa Mercadante de Assis}

Graduanda de Medicina na Universidade de Rio Verde (UniRV). Aparecida de Goiânia/ GO - Brasil. larissamercadante0@gmail.com

\section{Luisa Sousa Eleutério de Azevedo}

Graduanda de Medicina na Universidade de Rio Verde (UniRV). Aparecida de Goiânia/ GO - Brasil. luiisae25@gmail.com

\section{Nathalya Chaves Meirelles}

Graduanda de Medicina na Universidade de Rio Verde (UniRV). Aparecida de Goiânia/ GO - Brasil. naty_meirelles@hotmail.com

Submetido em: 24-8-2018

Aceito em: 10-6-2019 